\title{
Effects of feeding frequencies on the growth, plasma biochemistry, and liver glycogen of jade perch Scortum barcoo in a recirculating system
}

\begin{abstract}
Jade Perch Scortum barcoo having an initial average weight of $7.28 \pm 0.13 \mathrm{~g}$ (mean $\pm \mathrm{SE}$ ) were cultured in a recirculating system at different feeding frequencies that included one, two, three, or four meals a day at $5 \%$ body weight per day. Treatments were performed in triplicate using 65 fish in each replicate. After 72 d, the survival, growth, feeding efficiencies, body indices, plasma biochemistry, whole-body proximate composition, and muscle cholesterol were measured while histological sections of the liver were stained for glycogen with periodic acid Schiff. Results showed that growth was best when fish were fed three times a day, which was significantly higher than those fed one, two or four times a day. Feeding efficiencies were significantly better for fish fed two or three times a day. Plasma glucose, plasma cholesterol, periodic acid Schiff staining intensity of the liver, and hepatosomatic index were significantly higher for fish fed three times a day compared with once a day. Whole-body crude protein was significantly lower for fish fed once a day compared with treatments of two or four times a day. Muscle cholesterol tended to increase with increasing feeding frequencies, but the results were not significant. No significant treatment effect was detected on the plasma mineral content. Based on a feeding rate of $5 \%$ body weight/d, it is recommended that feedings are divided into three meals a day to increase available energy for optimal growth.
\end{abstract}

Keyword: Feeding frequencies; Recirculating system; Growth; Scortum barcoo 